\title{
Cartilage Oligomeric Matrix Protein Gene Multilayers Inhibit Osteogenic Differentiation and Promote Chondrogenic Differentiation of Mesenchymal Stem Cells
}

\author{
Peng Guo $^{\dagger}$, Zhong-Li Shi ${ }^{\dagger}$, An Liu, Tiao Lin, Fang-Gang Bi, Ming-Min Shi and Shi-Gui Yan ${ }^{1, *}$ \\ Department of Orthopaedic Surgery, the Second Affiliated Hospital, Medical College of Zhejiang \\ University, NO. 88 Jiefang Road, Hangzhou 310009, China; E-Mails: 21218130@zju.edu.cn (P.G.); \\ zlshi78@gmail.com (Z.-L.S.); liuan2013zju@gmail.com (A.L.); lintiao112009@gmail.com (T.L.); \\ fanggangbi@gmail.com (F.-G.B.); shimingmin@gmail.com (M.-M.S.)
}

$\dagger$ These authors contributed equally to this work.

* Author to whom correspondence should be addressed; E-Mail: zrjwsj@zju.edu.cn; Tel./Fax: +86-571-8778-3567.

External Editor: Emmanuel Stratakis

Received: 18 July 2014; in revised form: 22 September 2014 / Accepted: 27 October 2014 / Published: 5 November 2014

\begin{abstract}
There are still many challenges to acquire the optimal integration of biomedical materials with the surrounding tissues. Gene coatings on the surface of biomaterials may offer an effective approach to solve the problem. In order to investigate the gene multilayers mediated differentiation of mesenchymal stem cells (MSCs), gene functionalized films of hyaluronic acid (HA) and lipid-DNA complex (LDc) encoding cartilage oligomeric matrix protein (COMP) were constructed in this study via the layer-by-layer self-assembly technique. Characterizations of the HA/DNA multilayered films indicated the successful build-up process. Cells could be directly transfected by gene films and a higher expression could be obtained with the increasing bilayer number. The multilayered films were stable for a long period and DNA could be easily released in an enzymatic condition. Real-time polymerase chain reaction (RT-PCR) assay presented significantly higher $(p<0.01)$ COMP expression of MSCs cultured with HA/COMP multilayered films. Compared with control groups, the osteogenic gene expression levels of MSCs with HA/COMP multilayered films were down-regulated while the chondrogenic gene expression levels were up-regulated. Similarly, the alkaline phosphatase (ALP) staining and Alizarin red S staining of MSCs
\end{abstract}


with HA/COMP films were weakened while the alcian blue staining was enhanced. These results demonstrated that HA/COMP multilayered films could inhibit osteogenic differentiation and promote chondrogenic differentiation of MSCs, which might provide new insight for physiological ligament-bone healing.

Keywords: cartilage oligomeric matrix protein; layer-by-layer self-assembly; gene delivery; mesenchymal stem cells; cell differentiation

\section{Introduction}

Biomedical materials as medical devices have drawn great attention in both basic research and clinical application fields [1]. Surface modification of biomaterials (such as immobilization of the bioactive agents and chemical components), which can influence the manifestation and behavior of cells, has been demonstrated to be an efficient way to acquire a new nature for specific requirements [2-5]. In spite of this, the interface between implant and tissue is of great concern. Many efforts have been made to improve the integration of biomedical plants with the surrounding tissues which is vital for the success of various surgical procedures [6,7]. Considering the long-term efficiency of gene therapy, surface-mediated gene delivery is a promising method to promote the tissue ingrowth of the interface [8-11].

As first described by Decher et al. [12,13], the layer-by-layer (LbL) self-assembly technique has been widely used to construct ultrathin multilayers for gene transfection. The major advantage of the LbL technique is that it provides an approach to obtain controlled and sustaining release of DNA [14-16]. Although many studies [17-19] have focused on the osteogenic differentiation effects of multifarious gene films on mesenchymal stem cells (MSCs), there is still a lack of research on investigating the interactions for chondrogenic effects of gene films on MSCs.

A layer of calcified fibrocartilage is reported to be a major transition structure in the junction between bone and ligament [20]. This means it is important to sustain the cartilage layer in the interface between bone and ligament for physiological ligament-bone healing. Cartilage oligomeric matrix protein (COMP), being one of the most significant non-collagenous proteins in fibrous cartilage, belongs to the thrombospondin family and plays a vital role in the adhesion, proliferation and differentiation of cells [21-23]. Du YY et al. [24] found that COMP inhibited vascular smooth muscle cells calcification by interacting with bone morphogenetic protein 2 (BMP2). All these show that COMP has an important effect on cell differentiation.

Therefore, hyaluronic acid (HA)/COMP (lipid-DNA complex, LDc) multilayered films were constructed in the present study according to the HA/LDc fabrication method described before $[15,25]$. HA was chosen as a polyanion to interact with COMP-LDc because of its ideal biocompatibility and wide distribution in tissues. Multilayered films were presented as (HA/DNA) $(n$ is the number of bilayers).

The object of this study was to construct and characterize the multilayered films functionalized with pIRES-hrGFP-COMP gene via the LbL technique, and to investigate whether the gene-functionalized films possessed biological activity, as well as to determine what effect these gene films had on the differentiation of MSCs in vitro. 


\section{Results and Discussion}

\subsection{Characterization of the Multilayer Films}

\subsubsection{Contact Angle Measurement}

Contact angle measurement shows the wettability of the modified substrates, dependent on the hydrophilic properties of the outermost layers. A larger contact angle generally indicates a better hydrophilicity. The contact angle of the primal layers decreased compared with the bare substrates, which suggested improvement of hydrophilicity when the substrates were coated with chitosan (CHI) (Figure 1). Also the contact angle of the following layers undulated from $30^{\circ}$ to $10^{\circ}$ as alternate deposit of the HA and DNA layers. The fluctuant changes of the contact angle indicated alterations of the outermost layers, which confirmed alternate fabrication of the HA and DNA layers on the substrates.

Figure 1. Water contact angles as a function of the number of coating layers. The even layer numbers correspond to DNA layers, and the odd layer numbers correspond to hyaluronic acid (HA) layers $(n=9)$.

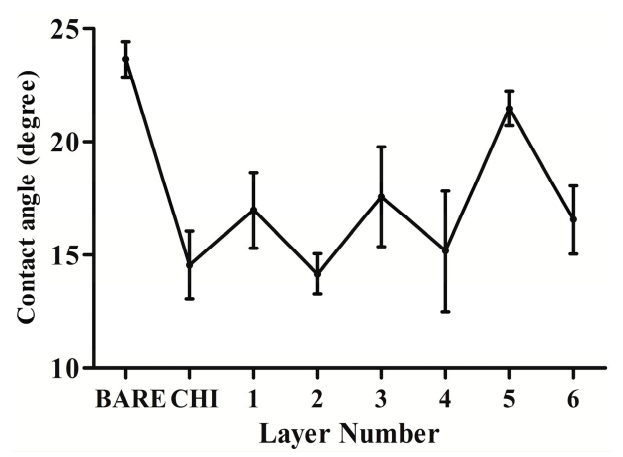

\subsubsection{UV-Vis Absorption}

UV-vis absorption at $260 \mathrm{~nm}$ is specifically contributed by the quantity of DNA, which thereby is a convenient way to monitor the build-up process by UV-vis spectrophotometer. The results of UV-vis absorbance at $260 \mathrm{~nm}$ of the multilayered DNA films are shown in Figure 2. The continuous increase of the UV-vis absorbance with the growth of bilayer number indicated that DNA was sequentially incorporated into the films, which also meant a successful fabrication process.

Figure 2. UV-vis absorbance of multilayered films at $260 \mathrm{~nm}$ as a function of layer number $(n=6)$.

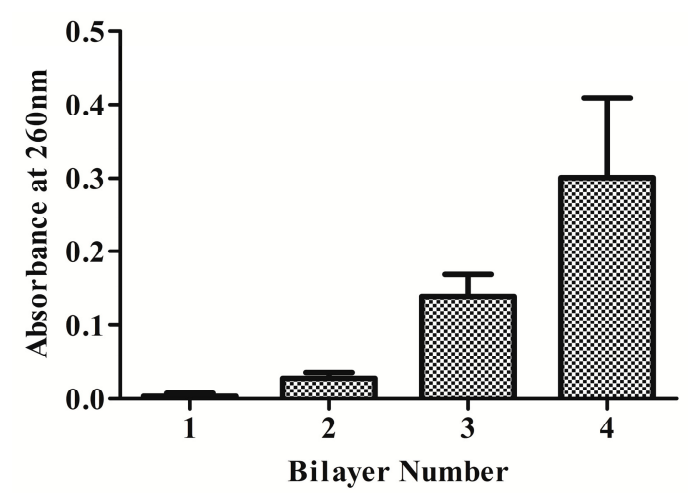




\subsubsection{Surface Morphology}

The morphology of the bare silicon surfaces, $(\mathrm{HA} / \mathrm{DNA})_{4}$ and (HA/DNA) 8 films under different magnification was characterized by scanning electron microscopy (SEM). The multilayered films displayed a quite rough facade, which was different from the smooth and uniform appearance on the bare surface (Figure 3). (HA/DNA)4 films exhibited loose fragmental surfaces while (HA/DNA)8 films showed compact particle-like microstructures.

Figure 3. Scanning electron microscopy (SEM) images of the bare surface (A), (HA/DNA)4 films (B) and (HA/DNA) 8 films (C); (a1), (b1) and (c1): 5000×; (a2), (b2) and (c2): 10,000×.

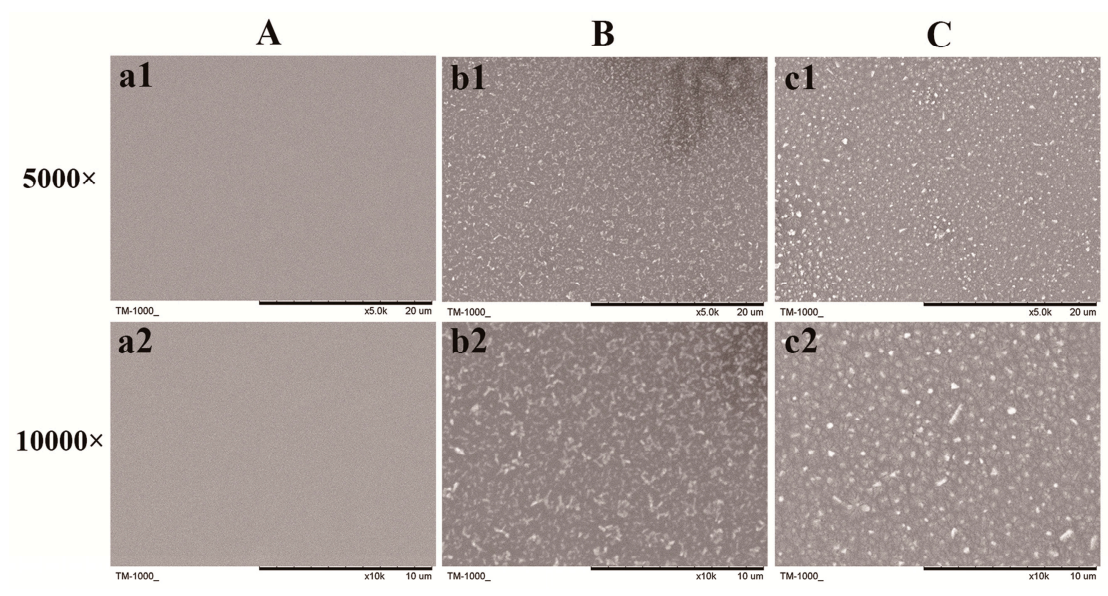

2.1.4. Stability Assay and Enzymatic Degradation of Multilayered Films

PBS buffer was chosen as the model of the physiological condition for the stability of (HA/DNA)6 films. As shown in Figure 4A, there was hardly any decrease of the amount of DNA in the films even after 10 days incubation. This result suggested that the films were stable in the hypothetic physiological situation for more than 10 days.

However, it was quite different when multilayered films were immersed in the trypsin solution. The DNA was released from (HA/DNA) 6 films to the incubation buffer, leading to a decrease of absorbance of the films and a remarkable increase of absorbance of the incubation buffer at day one (Figure 4B). This demonstrated that the enzymatic condition promoted the degradation process of multilayered films.

Figure 4. UV-vis absorbance of (HA/DNA)6 films and incubation buffer at $260 \mathrm{~nm}$ as function of time after incubation in (A) PBS at $37^{\circ} \mathrm{C}, \mathrm{pH} 7.4(n=3)$ and (B) the trypsin solution $\left(0.1 \mathrm{mg} / \mathrm{mL}\right.$ in $\mathrm{PBS}$ buffer) at $37^{\circ} \mathrm{C}$, pH $7.4(n=3)$.

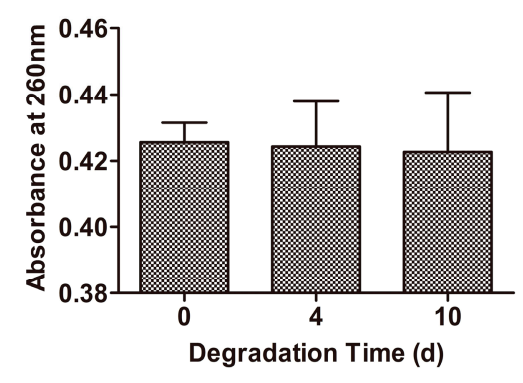

(A)

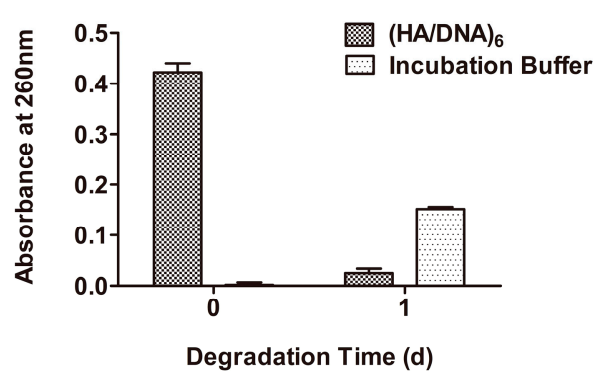

(B) 


\subsection{In Vitro Gene Transfection}

Figure 5 shows the typical images of the expression of GFP by HEK293T cells cultured on different substrates after three days transfection. Cells on tissue culture plates (TCPs) without transfection were used as negative control, while TCPs transfected with Lipofectamine ${ }^{\mathrm{TM}}$ LTX and PLUS reagent were used as positive control. The GFP expression indicated efficient transfection by the DNA films. Also the expression of GFP on (HA/DNA)6 films was significantly higher $(p<0.05)$ than those on (HA/DNA)2 according to the different fluorescence counts (Figure 5B). The results suggested that increasing the layer number would contribute to higher gene transfection efficiency after three days of culture.

Figure 5. (A) GFP expression of $293 \mathrm{~T}$ cells on different substrates after three days of culture. (a) (HA/DNA)2 films; (b) (HA/DNA)6 films; (c) tissue culture plates (TCPs) with Lipofectamine ${ }^{\mathrm{TM}}$ LTX and PLUS reagent, (d) TCPs. Scale bar: $200 \mu \mathrm{m}$; (B) The fluorescence counts of GFP expression in HEK293T cells after culturing on (HA/DNA) 2 and (HA/DNA) 6 films for three days $(n=5$, mean $\pm \mathrm{SD}, * p<0.05)$.

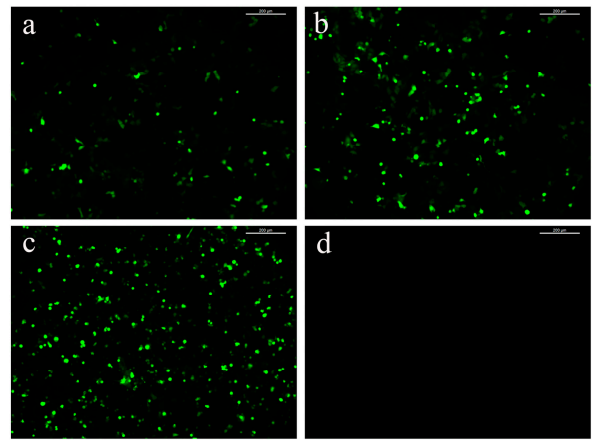

(A)

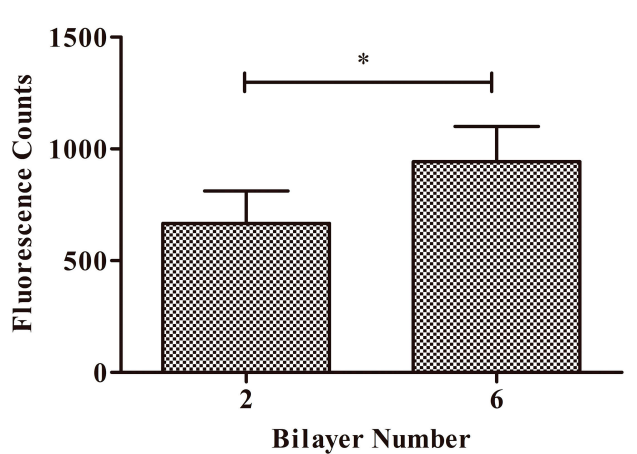

(B)

\subsection{Cartilage Oligomeric Matrix Protein (COMP) Expression Detection}

Real-time PCR assay was performed to confirm the expression level of COMP in MSCs using glyceraldehyde-3-phosphate dehydrogenase (GAPDH) as a reference gene for normalization. In this study, plasmid encoding recombinant human COMP was used and specific primers for human COMP was chosen for the real-time PCR. As shown in Figure 6, cells contacted with HA/COMP films obtained significantly higher $(p<0.01)$ expression of COMP than HA/pIRES films for more than 14 days. Although a gradual reduction of expression was observed, high expression of COMP lasted for more than 14 days.

\subsection{Osteogenic and Chondrogenic Gene Expression in Mesenchymal Stem Cells (MSCs)}

In order to investigate MSCs differentiation at the gene level, real-time PCR was performed to detect the mRNA expression level of related genes at day 3, 7 and 14. In this study, we selected collagen type I alpha 1 (Col1 $\alpha 1$ ), alkaline phosphatase (ALP), runt related transcription factor 2 (RUNX2), bone morphogenetic protein 2 (BMP2), osteopontin (OPN), bone gla protein (BGP) as osteogenic markers and collagen type II alpha 1 (Col2 $\alpha 1)$, aggrecan (ACAN), sry-related high-mobilitygroup box 9 (SOX9), collagen type $\mathrm{X}$ alpha 1 (Col10 $\alpha 1)$ as chondrogenic markers. 
HA/COMP films significantly $(p<0.01)$ down-regulated the mRNA expression of RUNX2 and BMP2 at most time points except for BMP2 at day 3 compared with HA/pIRES films (Figure 7). The functionalized gene films did not exert any significant effect on mRNA expression of ALP, OPN and BGP except for a decrease of OPN and an increase of BGP at day 3. A trend of down regulation of ALP and OPN by HA/COMP films appeared compared with HA/pIRES films but only the decreased expression of OPN at day 3 achieved statistical difference $(p<0.01)$ (Figure 7B,E). Although the expression level of Col1 $\alpha 1$ gene in MSCs cultured with HA/COMP films was enhanced at the early stage of osteogenic differentiation (day 3 and 7), a suppression was found after 14 days of culture (Figure 7A). The expression level of BGP in MSCs cultured with HA/COMP films were elevated at the early stage and then arrived at the same level at the late stage compared with HA/pIRES films (Figure 7F).

An opposite trend occurred when chondrogenic differentiation assay was performed (Figure 8). A consistent up-regulation of mRNA expression levels of Col2 $\alpha 1, A C A N$ and SOX9 for HA/COMP films were observed except for Col2 $\alpha 1$ at day 3. Also the expression of Col2 $\alpha 1$ increased gradually over time. However, Col10 $1 \mathrm{l}$ mRNA expression was only up-regulated in MSCs incubated with HA/COMP films at the late stage of chondrogenic differentiation (day 14).

Figure 6. The relative mRNA expression of human cartilage oligomeric matrix protein $(C O M P)$ was analyzed by real-time PCR at different time points. The value was normalized to $\operatorname{GAPDH}(n=3, * * p<0.01)$.

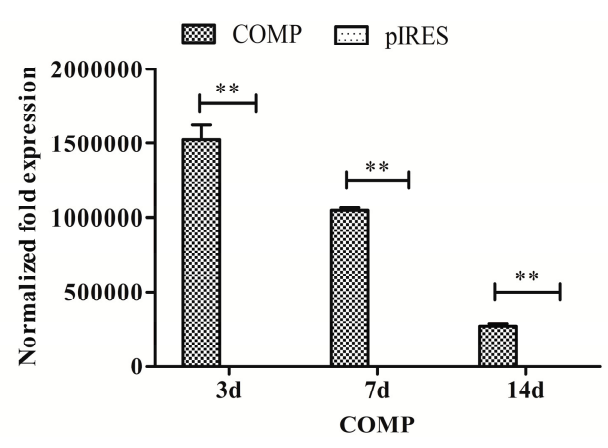

Figure 7. The relative mRNA expression of (A) collagen type I alpha 1 (Coll $\alpha 1)$; (B) alkaline phosphatase $(A L P)$; (C) runt related transcription factor $2(R U N X 2)$; (D) bone morphogenetic protein $2(B M P 2)$; (E) osteopontin $(O P N)$ and $(\mathbf{F})$ bone gla protein $(B G P)$ were analyzed by real-time PCR. The value was normalized to glyceraldehyde-3-phosphate dehydrogenase $(\mathrm{GAPDH})(n=3, * p<0.05, * * p<0.01)$.
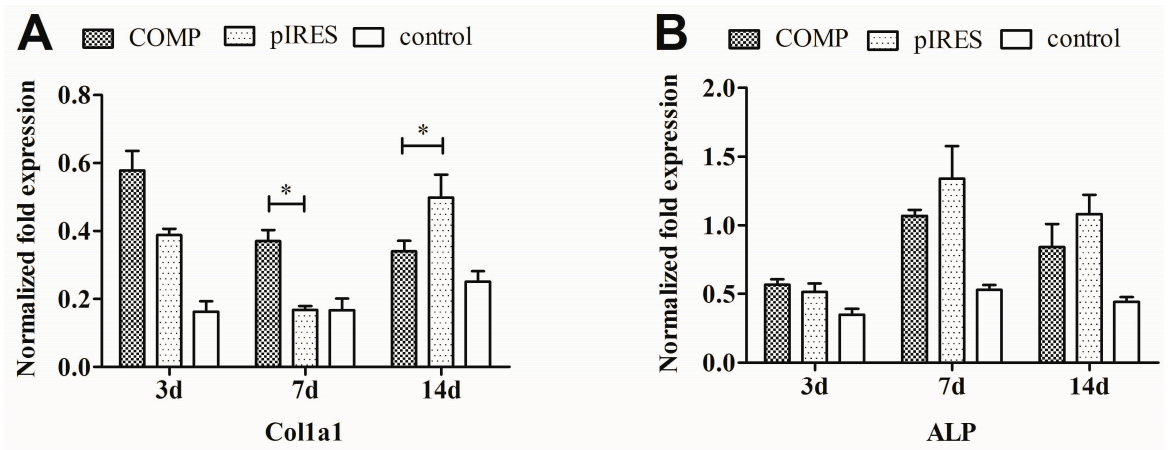
Figure 7. Cont.
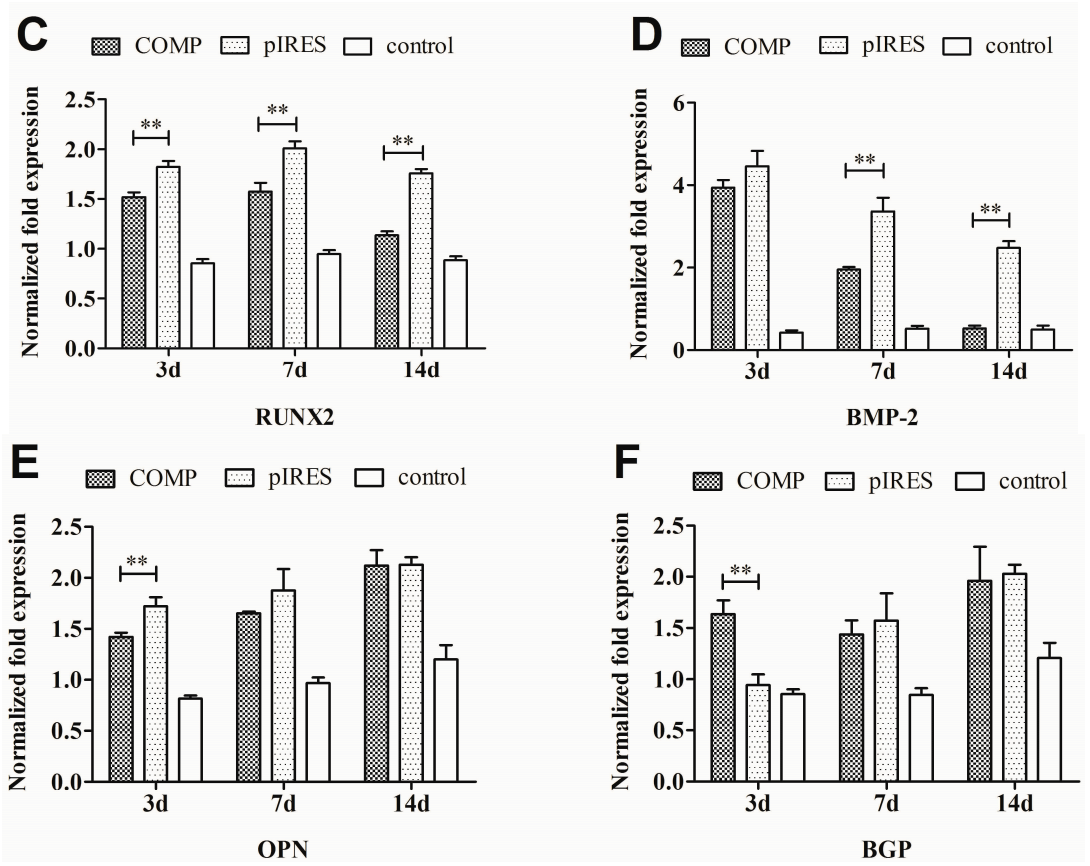

Figure 8. The relative mRNA expression of (A) collagen type II alpha 1 (Col2al); (B) aggrecan $(A C A N)$; (C) sry-related high-mobilitygroup box 9 (SOX9) and (D) collagen type X alpha 1 (Col10 $\alpha 1)$ were analyzed by real-time PCR. The value was normalized to GAPDH $\left(n=3,{ }^{*} p<0.05, * * p<0.01\right)$.

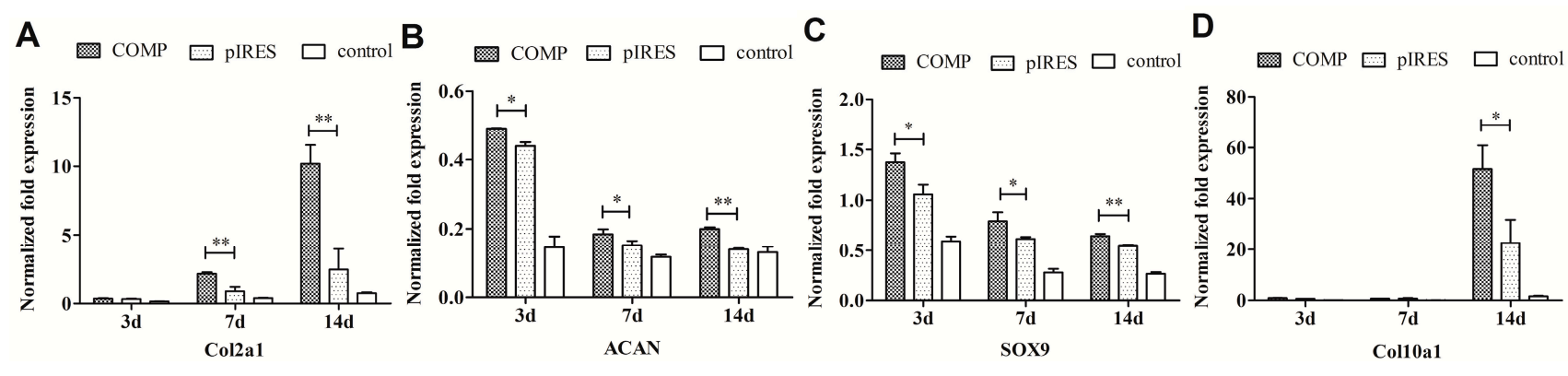

\subsection{Cell Staining}

To further investigate MSCs differentiation cultured with different multilayered films, cell staining was performed for osteogenic differentiation and chondrogenic differentiation.

Osteogenic differentiation was assessed by alkaline phosphatase (ALP) staining and alizarin red S staining. ALP activity was an early marker of osteogenesis. Also alizarin red S stained the calcium precipitation when mineralized matrix nodules were formed. ALP staining was conducted on day 7. In conformity to the gene expression levels above, both the intensity and the area of ALP staining decreased when MSCs were cultured with HA/COMP films compared with HA/pIRES films, which signified a lower ALP activity (Figure 9A). Alizarin red S staining was conducted at day 14 since calcium precipitation was formed at the late stage of osteogenesis differentiation. Similarly, alizarin red S staining of MSCs cultured with HA/COMP films was weakened according to the fewer number of mineralized nodules (Figure 9B). 
Chondrogenic differentiation was assessed by alcian blue staining for the proteoglycans at day 21 . In contrast with osteogenic staining, enhanced alcian blue staining of MSCs cultured with HA/COMP films indicated the accumulation of more proteoglycans. Also it agreed with the up regulation of chondrogenic gene expression levels (Figure 9C).

Figure 9. Cell staining of mesenchymal stem cells (MSCs) cultured with TCPs, HA/pIRES films and HA/COMP films. (A) alkaline phosphatase (ALP) staining of MSCs after osteogenic induction for 7 days; (B) Alizarin red S staining of MSCs after osteogenic induction for 14 days; (C) Alcian blue staining of MSCs after chondrogenic induction for 21 days.

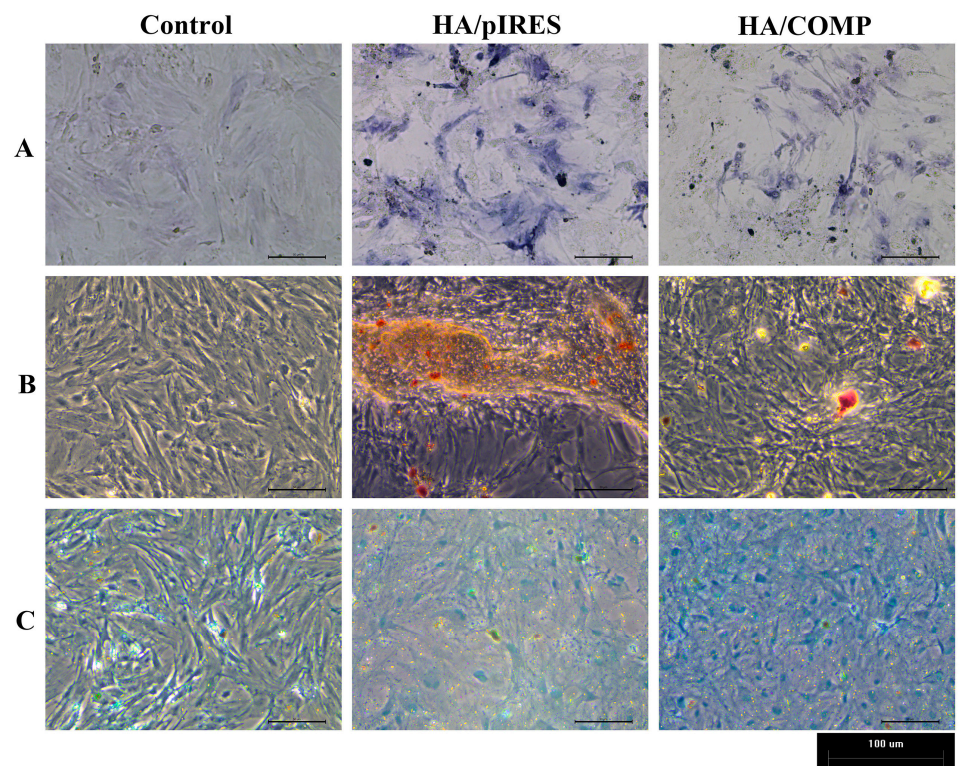

\subsection{Discussion}

Biological coatings have been applied to acquire ideal surface properties of medical devices in different situations. Immobilization of bioactive agents [5,26-29] and tuning mechanical properties of substrates [10,15,30-32] can efficiently direct cell behavior and fate. Deposit of gene components to modify substrates, resulting in a long-term efficiency, is one of the valued strategies $[18,19]$. As such, genes encoding growth factors such as vascular endothelial growth factor (VEGF) and BMP2 are utilized to promote osteogenic differentiation and bone formation [17,33]. However, gene-functionalized substrates for physiological ligament-bone healing have had lack of attention. As an important non-collagenous protein of the extracellular matrix (ECM) in fibrous cartilage, COMP plays a vital role in cell differentiation and the maintenance of cartilage phenotype [21-24,34,35].

Therefore, in this study, the plasmid DNA encoding COMP was employed to construct the gene-functionalized films through the $\mathrm{LbL}$ technique in consideration of its controlled and sustaining release. Successful construction with rough morphology of the HA/COMP multilayered films was monitored (Figures 1-3). The multilayered films were stable in physiological condition and the plasmid could be easily released in enzymatic condition (Figure 4), which agreed with previous studies [36,37]. There were various kinds of enzymes in vivo and trypsin was used as a typical one. This indicated that plasmid could be released from the multilayered films in vivo. The properties of stability 
and enzymatic degradation provide the basis of wide application in medical devices. Transfection assay of HEK293T cells demonstrated that the multilayered films could transfect cells and the GFP expression increased with the increasing layer number (Figure 5). This suggested transfection efficiency could be easily regulated by tuning the layer number. It was consistent with other reports $[15,37]$.

To investigate whether the target gene COMP could successfully express in MSCs through gene functionalized films, real-time PCR assay of COMP was carried out. A higher mRNA expression of COMP was detected in MSCs transfected with HA/COMP films compared with HA/pIRES films (Figure 6). It was reported that the expression level of exogenous gene transfected by liposome reaches a peak after three days culture and could not be sustained for a long time because of the loss of exogenous gene $[19,38,39]$. The high expression of COMP lasted for more than 14 days, which was much longer than the expression of exogenous gene transfected directly by liposome. This result indicated that the HA/COMP multilayered films could sustain a long-term biological effect for gene transfection.

To further investigate the effect of HA/COMP multilayered films on the osteogenic differentiation of MSCs, real-time PCR of osteoblast-related gene markers was employed. A recent research reported that COMP could bind directly to BMP-2 through the $C$-terminus, inhibit BMP2 receptor binding and block BMP2 osteogenic signaling [24]. BMP2 is known to induce MSCs osteogenic differentiation and stimulate ossification [40]. Meanwhile, RUNX2 is a key transcription factor for osteogenesis and the expression level of RUNX2 could be stimulated by BMP2 [41,42]. In the present study, BMP2 might be blocked by COMP, which could lead to the subsequent down-regulation of RUNX2. All these would contribute to the suppression of osteogenic process. As a result, most of osteoblast-related gene expression levels and osteogenic staining of MSCs cultured with HA/COMP films were restrained compared with HA/pIRES films. It could also be demonstrated by the weaken staining of ALP (Figure 9A). The expression levels of Coll $\alpha 1$ and $B G P$ were up-regulated at the early stage and then down-regulated or just flat at the late stage by HA/COMP films (Figure 7A,F). A hypothesis occurred to us that there might be a kind of negative feedback regulation. More research was needed since few studies have focused on this. Weakened alizarin red S staining also indicated the suppression of the mineralization of MSCs cultured with HA/COMP films (Figure 9B). All these suggested that the functionalized gene films could inhibit osteogenic differentiation of MSCs.

Meanwhile, chondrogenic differentiation assay was employed for real-time PCR and alcian blue staining. Col $2 \alpha 1$ is one of the most important molecular markers for chondrogenesis. The increasing expression of Col2al over time (Figure 8) indicated efficient chondrogenic differentiation and the up-regulated expression of Col2 $\alpha 1$ suggested the promotion of chondrogenic differentiation of MSCs by HA/COMP films. The expression levels of SOX9 and ACAN were also up-regulated, which was in accordance with the enhanced alcian blue staining by HA/COMP films (Figure 9C).

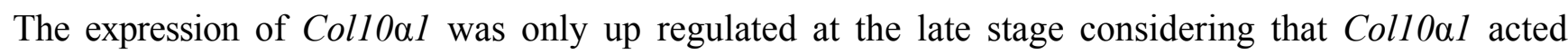
as a late markerof chondrogenic differentiation and it was thought to be an indicator for the maturity and hypertrophy of chondrocytes. COMP is a signaling protein including many domains, such as its $C$-terminals, RGD domains and T3C5 domains [22,23]. Also as a kind of integrin ligand, COMP can recognize and bond integrin $\alpha 5 \beta 1$ and start the following signal transduction to direct cell behavior and fate [21]. The results revealed that the functionalized gene films could promote chondrogenic differentiation of MSCs. Altogether, we confirmed that COMP gene multilayers could inhibit osteogenic differentiation and promote chondrogenic differentiation of MSCs in vitro. 
There are some limitations in our study. As a preliminary study, this study focused on the effect of multilayered COMP films on the differentiation of MSCs in vitro. Effects of the multilayered films in vivo for ligament-bone healing could not be observed. In a future study, the combination of exogenous MSCs and implants coated with multilayered COMP films for ligament-bone healing in vivo will be investigated.

\section{Experimental Section}

\subsection{Materials}

Chitosan (CHI) and hyaluronic acid (HA) were purchased from Sigma Chemical Co. (St. Louis, MO, USA). The pIRES-hrGFP-1a plasmid encoding recombinant human COMP was provided by Sangon Biotech (Shanghai, China). HEK293T cell line was obtained from American type culture collection (ATCC, Shanghai, China). Silicon wafers $(20 \mathrm{~mm} \times 20 \mathrm{~mm}, 1.5 \mathrm{~mm}$ thickness; commercially double polishing wafer) and quartz were kindly provided by Guangci Medical Device Co. Ltd. (Hangzhou, China). Glass coverslips $(\varphi=14 \mathrm{~mm})$ were purchased from NEST (Wuxi, China). Mesenchymal stem cells (MSCs), corresponding stem cell products and cell staining reagents were provided by Cyagen (Guangzhou, China) except for special note. Information of the components of the osteogenic (Catalog NO. RASMX-90021) and chondrogenic (Catalog NO. RASMX-90041) media can be obtained on the Cyagen official website.

\subsection{DNA Complex Preparation}

pIRES-hrGFP-1a (pIRES) or pIRES-hrGFP-1a-COMP (COMP) plasmid DNA complex was prepared using Lipofectamine ${ }^{\mathrm{TM}}$ LTX and PLUS reagent (Invitrogen, Carlsbad, CA, USA) according to the manufacturer's instruction. In brief, $100 \mu \mathrm{g}$ of plasmid DNA was diluted with Opti-MEM (Life Technologies, Carlsbad, CA, USA) and mixed with $100 \mu \mathrm{L}$ PLUS reagent and $300 \mu \mathrm{L}$ LTX reagent to a final volume of $10 \mathrm{~mL}$ (LDc solution). Then the mixture was incubated at room temperature for $30 \mathrm{~min}$ before fabrication.

\subsection{Pretreatment of Substrates}

Silicon wafers, quartz substrates and glass coverslips chosen for the fabrication were cleaned following the procedure for multilayered films fabrication as described by Wang, X.F. et al. [43]. Namely, the substrate was cleaned sequentially in methanol, ethanol, methanol and water, immersed in a Piranha solution (7:3 (vol. \%), 98\% $\mathrm{H}_{2} \mathrm{SO}_{4}: 30 \% \mathrm{H}_{2} \mathrm{O}_{2}$ ) for $10 \mathrm{~min}$, and then 1:1:5 (vol. \%), $30 \% \mathrm{H}_{2} \mathrm{O}_{2}: 25 \% \mathrm{NH}_{3}: \mathrm{H}_{2} \mathrm{O}$ at $60{ }^{\circ} \mathrm{C}$ for $30 \mathrm{~min}$, finally washed with deionized water and dried at room temperature before use. All these chemical reagents were provided by Sinopharm Chemical Reagent Co., Ltd. (Shanghai, China).

\subsection{Fabrication of Multilayered Films}

CHI solution $(5 \mathrm{mg} / \mathrm{mL}$ ) was prepared with $2 \%$ (vol. \%) acetic acid and HA was dissolved in distilled water with a concentration of $0.5 \mathrm{mg} / \mathrm{mL}$. The substrates were originally dipped in CHI solution 
for $30 \mathrm{~min}$ to form a precursor layer with stable positive charge to initiate the following procedure. Then the (HA/DNA)n ( $n$ is the number of bilayer) multilayered films were fabricated by immersing substrates in HA solution for $10 \mathrm{~min}$. After being washed twice with Opti-MEM for $1 \mathrm{~min}$, substrates were deposited in LDc solution for $10 \mathrm{~min}$ and washed again. These procedures were repeated until the desired multilayered films were fabricated (Scheme 1). (HA/COMP) $n$ films were used in the follow-up characterization and cell experiments. Also (HA/pIRES)n films were chosen as negative control in cell experiments.

Scheme 1. Layer-by-layer assembly of (HA/DNA)n films. Substrate was first coated with Chitosan (CHI) and submerged in HA solution. Following a rinse in Opti-MEM twice, the substrate was then immersed in the lipid-DNA complex (LDc) solution. Another rinse was conducted before the next round.

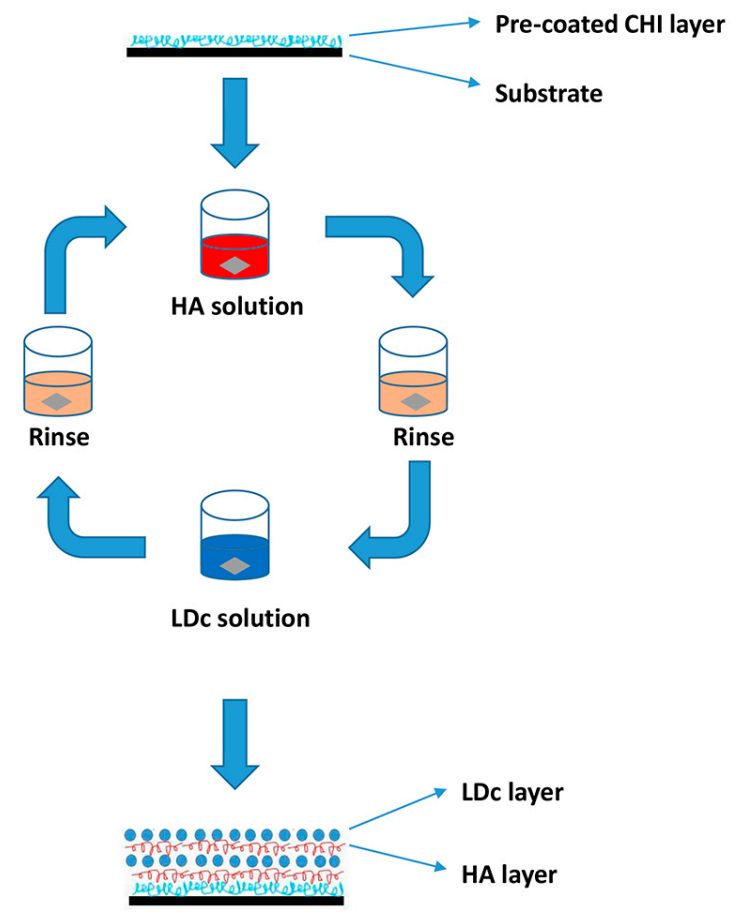

\subsection{Characterization of the Multilayered Films}

The build-up process of films was monitored through contact angle measurement by a video-based contact angle measuring device (OCA 20, Dataphysics, Stuttgart, Germany). The images of water drops on the surface were captured and analyzed following the instructions. Three samples in each group were chosen and three different points in each sample were measured.

The progressive buildup of films was also measured on a UV-vis spectrophotometer (Bio-Rad, Smart Spec ${ }^{\mathrm{TM}}$ Plus, Hercules, CA, USA). The UV-vis absorption at $260 \mathrm{~nm}$ of multilayered films on quartz slides was detected. Six samples in each group were used.

The morphology of multilayered films on silicon wafers was determined by a scanning electron microscopy (SEM; Hitachi, TM-1000, Tokyo, Japan). Samples were dried at room temperature after fabrication and sputter-coated with gold before detection. 
Films with six bilayers of HA/COMP were selected for the stability assay. The multilayered films were exposed to PBS buffer ( $\mathrm{pH}=7.4$, Life Technologies, Carlsbad, CA, USA) at $37{ }^{\circ} \mathrm{C}$. At appropriate time intervals, films were taken out, washed with deionized water, and dried before the measurement of UV-vis absorption at $260 \mathrm{~nm}$. Three samples were chosen as replicates.

Trypsin was chosen as the model protease to investigate the degradation properties of (HA/COMP) 6 films for the enzymatic degradation assay. The degradation samples were incubated in a trypsin solution $(0.1 \mathrm{mg} / \mathrm{mL}$ in PBS buffer, $\mathrm{pH}=7.4)$ at $37{ }^{\circ} \mathrm{C}$. The films were taken out of the incubation solution at desired time intervals and washed with PBS buffer before UV-vis absorption detection using a UV-vis spectrophotometer at the wavelength of $260 \mathrm{~nm}$. Also the incubation solution was measured at the same time. Three replicates were used as above.

\subsection{In Vitro Gene Transfection}

To assess the biological activity of the multilayered films, the (HA/COMP $)_{n}(n=2$ or 6$)$ films were fabricated on glass coverslips and $293 \mathrm{~T}$ cells were selected for the transfection experiment. The $293 \mathrm{~T}$ cells were seeded (density $1 \times 10^{4}$ cells $/ \mathrm{cm}^{2}$ ) into 24 -well plates (TCPs) to which multilayered films had been added in dulbecco's modified eagle medium (DMEM, Life Technologies, Carlsbad, CA, USA) at $37{ }^{\circ} \mathrm{C}$ and $5 \% \mathrm{CO}_{2}$. Cells in TCPs without multilayered films were selected as control and transfected with Lipofectamine ${ }^{\mathrm{TM}}$ LTX and PLUS reagent according to the manufacturer's instructions if mentioned. After incubation for three days, GFP expression of 293T cells was observed under a fluorescence microscope (Leica, DMI4000B, Solms, Germany). Fluorescence counts of different films were calculated by Image-Pro Plus 6.0 (Media Cybernetics, Rockville, MD, USA). The mean value of five replicates was used as the final result for each group.

\subsection{Transfection and Differentiation of MSCs}

The (HA/COMP)6 and (HA/pIRES)6 films were chosen in this study for the following cell experiments. MSCs were seeded (initial density $2 \times 10^{4}$ cells $/ \mathrm{cm}^{2}$ ) into 24-well plates in MSC growth medium for $4 \mathrm{~h}$ adhesion, after which the selected films were gently put on top of the cells. Then cells were cultured for $48 \mathrm{~h}$, followed by the process of MSC differentiation by replacing the growth medium with osteogenic or chondrogenic differentiation medium. Cells were harvested at appropriate time intervals for subsequent analysis. For COMP expression detection, growth medium was sustained till the end of the assay and cells were collected at desired time points for real-time PCR analysis.

\subsection{RNA Isolation and Real-Time PCR}

The total RNA of MSCs harvested at specified time points was extracted using TRIZOL reagent (Invitrogen, Carlsbad, CA, USA) according to the manufacturer's protocol. Reverse transcription reactions were carried out using Prime Script RT Reagent Kit (Takara, Dalian, China) according to the manufacturer's instructions. Real-time PCR was performed using SYBR Premix Ex Taq (Takara, Dalian, China) according to the manufacturer's instructions. Target genes were normalized to GAPDH expression and all sample values were calculated by the $2^{-\Delta \Delta C t}$ method. Table 1 shows the primer sequences used in this study. 
Table 1. Primer oligonucleotide sequences in this study.

\begin{tabular}{|c|c|c|}
\hline Gene & Oligonucleotides $\left(5^{\prime}-3^{\prime}\right)$ & Gene Bank ID \\
\hline \multirow{2}{*}{$G A P D H$} & F: GACATGCCGCCTGGAGAAAC & \multirow{2}{*}{ NM_017008.4 } \\
\hline & R: AGCCCAGGATGCCCTTTAGT & \\
\hline \multirow{2}{*}{$R U N X 2$} & F: GCGTCCTATCAGTTCCCAAT & \multirow{2}{*}{ NM_001278483.1 } \\
\hline & R: CAGCGTCAACACCATCATTC & \\
\hline \multirow{2}{*}{$O P N$} & F: CTTGGCTTACGGACTGAGG & \multirow{2}{*}{ NM_012881 } \\
\hline & R: GCAACTGGGATGACCTTGAT & \\
\hline \multirow{2}{*}{$B M P 2$} & F: TGAGGATTAGCAGGTCTTTGC & \multirow{2}{*}{ NM_017178.1 } \\
\hline & R: TCTCGTTTGTGGAGTGGATG & \\
\hline \multirow{2}{*}{$B G P$} & F: CAAGTCCCACACAGCAACTC & \multirow{2}{*}{ NM_013414.1 } \\
\hline & R: CCAGGTCAGAGAGGCAGAAT & \\
\hline \multirow{2}{*}{ COMP } & F: CCCAACTCAGACCAGAAGGA & \multirow{2}{*}{ NM_000095.2 } \\
\hline & R: GTCACAAGCATCTCCCACAA & \\
\hline \multirow{2}{*}{ SOX9 } & F: GACGTGCAAGCTGGGAAAGT & \multirow{2}{*}{ XM_001081628.3 } \\
\hline & R: CGGCAGGTATTGGTCAAACTC & \\
\hline \multirow{2}{*}{ Col $2 \alpha 1$} & F: CGCCACGGTCCTACAATGTC & \multirow{2}{*}{ NM_012929.1 } \\
\hline & R: GTCACCTCTGGGTCCTTGTTCAC & \\
\hline \multirow{2}{*}{$A C A N$} & F: TGGCATTGAGGACAGCGAAG & \multirow{2}{*}{ NM_022190.1 } \\
\hline & R: TCCAGTGTGTAGCGTGTGGAAATAG & \\
\hline \multirow{2}{*}{ Col10al } & F: GGGATGCCTCTTGTCAGTGC & \multirow{2}{*}{ XM_001053056.4 } \\
\hline & R: ATCTTGGGTCATAGTGCTGCTG & \\
\hline \multirow{2}{*}{ Coll $\alpha 1$} & F: GACATGTTCAGCTTTGTGGACCTC & \multirow{2}{*}{ NM_053304.1 } \\
\hline & R: GGGACCCTTAGGCCATTGTGTA & \\
\hline
\end{tabular}

\subsection{Cell Staining}

The cells cultured in osteogenic differentiation medium for seven days were stained using BCIP/NBT Alkaline Phosphatase Color Development Kit (Boster, Wuhan, China) for alkaline phosphatase (ALP) staining according to the protocol.

On day 14 after osteogenic differentiation, alizarin red $\mathrm{S}$ staining for calcium precipitation was performed as described before [44,45]. Briefly, cells were fixed with $4 \%$ formaldehyde solution for $30 \mathrm{~min}$, followed by washing twice with PBS. Then cells were stained with alizarin red S for $5 \mathrm{~min}$. After rinsing with PBS twice again, the staining of mineralized nodules was observed under a light microscope (Leica, DMIL, Solms, Germany).

On day 21 after chondrogenic differentiation, alcian blue staining for proteoglycans synthesized by chondrocytes was performed. Cells were washed with PBS, fixed with 4\% formaldehyde solution (Sinopharm Chemical Reagent Co., Ltd., Shanghai, China) for $30 \mathrm{~min}$ and washed again with PBS. Cells were stained with alcian blue solution (Cyagen, Guangzhou, China) for $30 \mathrm{~min}$ and washed with distilled water (Life Technologies, Carlsbad, CA, USA). Then cells were observed under a light microscope. 


\subsection{Statistical Analysis}

The results were expressed as mean \pm standard deviation (SD). An unpaired Student's $t$-test was performed to evaluate the significance of the observed differences between the study groups and a value of $p<0.05$ was considered to be statistically significant.

\section{Conclusions}

In summary, we developed an efficient COMP gene delivery system to regulate cell differentiation in this study. COMP plasmid DNA was successfully incorporated into multilayered films with HA via the LbL technique. Multilayered films, which were demonstrated to possess the property to transfect MSCs and induce continuous expression of COMP for at least two weeks, could inhibit osteogenic differentiation and promote chondrogenic differentiation of MSCs in vitro. The combination of surface modification and functional gene therapy provides a promising strategy for potential applications in the field of medical devices for physiological ligament-bone healing.

\section{Acknowledgments}

This work is supported by the National Natural Science Foundation of China $(81101377,81101345$, 81171687, 81371954, 81201414, 81201416), the key project of Zhejiang Provincial Department of science and technology (2011C13033), Zhejiang Provincial Natural Science Foundation of China (Y2100161, Y2090283), Scientific Research Fund of Zhejiang Provincial Education Department (Y201018936) and Fund of Health Department of Zhejiang province (2012RCA032).

\section{Author Contributions}

Peng Guo, Zhong-Li Shi and Shi-Gui Yan participated in the design and coordination of the study, and drafted the manuscript. Peng Guo, An Liu, Tiao Lin, Fang-Gang Bi and Ming-Min Shi performed the research. Peng Guo, Fang-Gang Bi and Ming-Min Shi participated in the data collection, analysis and interpretation. Peng Guo, An Liu and Tiao Lin carried out the samples collection and detection. Peng Guo, Zhong-Li Shi and Shi-Gui Yan participated in the revision of manuscript content. All authors read and approved the final manuscript.

\section{Conflicts of Interest}

The authors declare no conflict of interest.

\section{References}

1. Williams, D.F. On the nature of biomaterials. Biomaterials 2009, 30, 5897-5909.

2. Samuel, R.E.; Shukla, A.; Paik, D.H.; Wang, M.X.; Fang, J.C.; Schmidt, D.J.; Hammond, P.T. Osteoconductive protamine-based polyelectrolyte multilayer functionalized surfaces. Biomaterials 2011, 32, 7491-7502. 
3. Liang, M.; Yao, J.; Chen, X.; Huang, L.; Shao, Z. Silk fibroin immobilization on poly(ethylene terephthalate) films: Comparison of two surface modification methods and their effect on mesenchymal stem cells culture. Mater. Sci. Eng. 2013, 33, 1409-1416.

4. Holmes, C.A.; Tabrizian, M. Substrate-mediated gene delivery from glycol-chitosan/hyaluronic acid polyelectrolyte multilayer films. Appl. Mater. Interf. 2013, 5, 524-531.

5. Ji, J.; Zhu, H.; Shen, J. Surface tailoring of poly(D,L-lactic acid) by ligand-tethered amphiphilic polymer for promoting chondrocyte attachment and growth. Biomaterials 2004, 25, 1859-1867.

6. Petrie, T.A.; Raynor, J.E.; Reyes, C.D.; Burns, K.L.; Collard, D.M.; García, A.J. The effect of integrin-specific bioactive coatings on tissue healing and implant osseointegration. Biomaterials 2008, 29, 2849-2857.

7. Roach, P.; Eglin, D.; Rohde, K.; Perry, C.C. Modern biomaterials: A review-Bulk properties and implications of surface modifications. J. Mater. Sci. 2007, 18, 1263-1277.

8. Cai, K.; Hu, Y.; Luo, Z.; Kong, T.; Lai, M.; Sui, X.; Wang, Y.; Yang, L.; Deng, L. Cell-specific gene transfection from a gene-functionalized poly(D,L-lactic acid) substrate fabricated by the layer-by-layer assembly technique. Angew. Chem. Int. Ed. 2008, 47, 7479-7481.

9. Jessel, N.; Oulad-Abdelghani, M.; Meyer, F.; Lavalle, P.; Haîkel, Y.; Schaaf, P.; Voegel, J.-C. Multiple and time-scheduled in situ DNA delivery mediated by $\beta$-cyclodextrin embedded in a polyelectrolyte multilayer. Proc. Natl. Acad. Sci. USA 2006, 103, 8618-8621.

10. Kong, H.J.; Liu, J.; Riddle, K.; Matsumoto, T.; Leach, K.; Mooney, D.J. Non-viral gene delivery regulated by stiffness of cell adhesion substrates. Nat. Mater. 2005, 4, 460-464.

11. Shen, H.; Tan, J.; Saltzman, W.M. Surface-mediated gene transfer from nanocomposites of controlled texture. Nat. Mater. 2004, 3, 569-574.

12. Decher, G.; Hong, J.D.; Schmitt, J. Buildup of ultrathin multilayer films by a self-assembly process: III. Consecutively alternating adsorption of anionic and cationic polyelectrolytes on charged surfaces. Thin Solid Films 1992, 210-211, 831-835.

13. Decher, G. Fuzzy nanoassemblies: Toward layered polymeric multicomposites. Science 1997, 277, 1232-1237.

14. Ren, K.; Wang, Y.; Ji, J.; Lin, Q.; Shen, J. Construction and deconstruction of PLL/DNA multilayered films for DNA delivery: Effect of ionic strength. Colloids Surf. B 2005, 46, 63-69.

15. Liu, L.; Song, L.-N.; Yang, G.-L.; Zhao, S.-F.; He, F.-M. Fabrication, characterization, and biological assessment of multilayer DNA coatings on sandblasted-dual acid etched titanium surface. J. Biomed. Mater. Res. 2011, 97, 300-310.

16. Ren, K.; Ji, J.; Shen, J. Tunable DNA release from cross-linked ultrathin DNA/PLL multilayered films. Bioconjug. Chem. 2006, 17, 77-83.

17. Jiang, Q.-H.; Liu, L.; Shen, J.-W.; Peel, S.; Yang, G.-L.; Zhao, S.-F.; He, F.-M. Influence of multilayer rhBMP-2 DNA coating on the proliferation and differentiation of MC3T3-E1 cells seeded on roughed titanium surface. J. Biomed. Mater. Res. 2012, 100, 2766-2774.

18. Hu, Y.; Cai, K.; Luo, Z.; Zhang, Y.; Li, L.; Lai, M.; Hou, Y.; Huang, Y.; Li, J.; Ding, X.; et al. Regulation of the differentiation of mesenchymal stem cells in vitro and osteogenesis in vivo by microenvironmental modification of titanium alloy surfaces. Biomaterials 2012, 33, 3515-3528. 
19. Hu, Y.; Cai, K.; Luo, Z.; Zhang, R.; Yang, L.; Deng, L.; Jandt, K.D. Surface mediated in situ differentiation of mesenchymal stem cells on gene-functionalized titanium films fabricated by layer-by-layer technique. Biomaterials 2009, 30, 3626-3635.

20. Yang, P.J.; Temenoff, J.S. Engineering orthopedic tissue interfaces. Tissue Eng. 2009, 15, 127-141.

21. Rock, M.J.; Holden, P.; Horton, W.A.; Cohn, D.H. Cartilage oligomeric matrix protein promotes cell attachment via two independent mechanisms involving CD47 and $\alpha \mathrm{V} \beta 3$ integrin. Mol. Cell. Biochem. 2010, 338, 215-224.

22. Tan, K.; Duquette, M.; Joachimiak, A.; Lawler, J. The crystal structure of the signature domain of cartilage oligomeric matrix protein: Implications for collagen, glycosaminoglycan and integrin binding. FASEB J. 2009, 23, 2490-2501.

23. Chen, F.H.; Herndon, M.E.; Patel, N.; Hecht, J.T.; Tuan, R.S.; Lawler, J. Interaction of cartilage oligomeric matrix protein/thrombospondin 5 with aggrecan. J. Biol. Chem. 2007, 282, 24591-24598.

24. Du, Y.; Wang, Y.; Wang, L.; Liu, B.; Tian, Q.; Liu, C.-J.; Zhang, T.; Xu, Q.; Zhu, Y.; Ake, O.; et al. Cartilage oligomeric matrix protein inhibits vascular smooth muscle calcification by interacting with bone morphogenetic protein-2. Circ. Res. 2011, 108, 917-928.

25. Yamauchi, F.; Koyamatsu, Y.; Kato, K.; Iwata, H. Layer-by-layer assembly of cationic lipid and plasmid DNA onto gold surface for stent-assisted gene transfer. Biomaterials 2006, 27, 3497-3504.

26. Suci, P.A.; Klem, M.T.; Arce, F.T.; Douglas, T.; Young, M. Assembly of multilayer films incorporating a viral protein cage architecture. Langmuir 2006, 22, 8891-8896.

27. Li, G.; Yang, P.; Qin, W.; Maitz, M.F.; Zhou, S.; Huang, N. The effect of coimmobilizing heparin and fibronectin on titanium on hemocompatibility and endothelialization. Biomaterials 2011, 32, 4691-4703.

28. Lin, Q.; Ding, X.; Qiu, F.; Song, X.; Fu, G.; Ji, J. In situ endothelialization of intravascular stents coated with an anti-CD34 antibody functionalized heparin-collagen multilayer. Biomaterials 2010, $31,4017-4025$.

29. Zan, X.; Sitasuwan, P.; Powell, J.; Dreher, T.W.; Wang, Q. Polyvalent display of RGD motifs on turnip yellow mosaic virus for enhanced stem cell adhesion and spreading. Acta Biomater. 2012, 8, 2978-2985.

30. Richert, L.; Schneider, A.; Vautier, D.; Vodouhe, C.; Jessel, N.; Payan, E.; Schaaf, P.; Voegel, J.-C.; Picart, C. Imaging cell interactions with native and crosslinked polyelectrolyte multilayers. Cell Biochem. Biophys. 2006, 44, 273-285.

31. Blin, G.; Lablack, N.; Louis-Tisserand, M.; Nicolas, C.; Picart, C.; Pucéat, M. Nano-scale control of cellular environment to drive embryonic stem cells selfrenewal and fate. Biomaterials 2010, 31 , $1742-1750$.

32. Blacklock, J.; Vetter, A.; Lankenau, A.; Oupický, D.; Möhwald, H. Tuning the mechanical properties of bioreducible multilayer films for improved cell adhesion and transfection activity. Biomaterials 2010, 31, 7167-7174.

33. Keeney, M.; van den Beucken, J.J.J.P.; van der Kraan, P.M.; Jansen, J.A.; Pandit, A. The ability of a collagen/calcium phosphate scaffold to act as its own vector for gene delivery and to promote bone formation via transfection with VEGF165. Biomaterials 2010, 31, 2893-2902.

34. Milz, S.; Aktas, T.; Putz, R.; Benjamin, M. Expression of extracellular matrix molecules typical of articular cartilage in the human scapholunate interosseous ligament. J. Anat. 2006, 208, 671-679. 
35. Zaucke, F.; Grässel, S. Genetic mouse models for the functional analysis of the perifibrillar components collagen IX, COMP and matrilin-3: Implications for growth cartilage differentiation and endochondral ossification. Histol. Histopathol. 2009, 24, 1067-1079.

36. Ren, K.; Ji, J.; Shen, J. Construction and enzymatic degradation of multilayered poly-l-lysine/DNA films. Biomaterials 2006, 27, 1152-1159.

37. Chang, H.; Ren, K.-F.; Wang, J.-L.; Zhang, H.; Wang, B.-L.; Zheng, S.-M.; Zhou, Y.-Y.; Ji, J. Surface-mediated functional gene delivery: An effective strategy for enhancing competitiveness of endothelial cells over smooth muscle cells. Biomaterials 2013, 34, 3345-3354.

38. Larchian, W.A.; Horiguchi, Y.; Nair, S.K.; Fair, W.R.; Heston, W.D.; Gilboa, E. Effectiveness of combined interleukin 2 and B7.1 vaccination strategy is dependent on the sequence and order: A liposome-mediated gene therapy treatment for bladder cancer. Clin. Cancer Res. 2000, 6, 2913-2920.

39. Tanswell, A.K.; Staub, O.; Iles, R.; Belcastro, R.; Cabacungan, J.; Sedlackova, L.; Steer, B.; Wen, Y.; $\mathrm{Hu}$, J.; O’Brodovich, H. Liposome-mediated transfection of fetal lung epithelial cells: DNA degradation and enhanced superoxide toxicity. Am. J. Phys. 1998, 275, L452-L460.

40. Lian, J.B.; Stein, G.S.; Javed, A.; van Wijnen, A.J.; Stein, J.L.; Montecino, M.; Hassan, M.Q.; Gaur, T.; Lengner, C.J.; Young, D.W. Networks and hubs for the transcriptional control of osteoblastogenesis. Rev. Endocr. Metab. Disord. 2006, 7, 1-16.

41. Lee, K.S.; Kim, H.J.; Li, Q.L.; Chi, X.Z.; Ueta, C.; Komori, T.; Wozney, J.M.; Kim, E.G.; Choi, J.Y.; Ryoo, H.M.; et al. Runx2 is a common target of transforming growth factor $\beta 1$ and bone morphogenetic protein 2, and cooperation between Runx2 and Smad5 induces osteoblast-specific gene expression in the pluripotent mesenchymal precursor cell line $\mathrm{C} 2 \mathrm{C} 12$. Mol. Cell. Biol. 2000, 20, 8783-8792.

42. Lee, M.H.; Javed, A.; Kim, H.J.; Shin, H.I.; Gutierrez, S.; Choi, J.Y.; Rosen, V.; Stein, J.L.; van Wijnen, A.J.; Stein, G.S.; et al. Transient upregulation of CBFA1 in response to bone morphogenetic protein-2 and transforming growth factor $\beta 1$ in $\mathrm{C} 2 \mathrm{C} 12$ myogenic cells coincides with suppression of the myogenic phenotype but is not sufficient for osteoblast differentiation. J. Cell. Biochem. 1999, 73, 114-125.

43. Wang, X.; Sun, J.; Ji, J. pH modulated layer-by-layer assembly as a new approach to tunable formulating of DNA within multilayer coating. React. Funct. Polym. 2011, 71, 254-260.

44. Sharff, K.A.; Song, W.-X.; Luo, X.; Tang, N.; Luo, J.; Chen, J.; Bi, Y.; He, B.-C.; Huang, J.; Li, X.; et al. Heyl basic helix-loop-helix protein plays an important role in mediating BMP9-induced osteogenic differentiation of mesenchymal progenitor cells. J. Biol. Chem. 2009, 284, 649-659.

45. Liao, J.; Hu, N.; Zhou, N.; Lin, L.; Zhao, C.; Yi, S.; Fan, T.; Bao, W.; Liang, X.; Chen, H.; et al. Sox9 potentiates BMP2-induced chondrogenic differentiation and inhibits BMP2-induced osteogenic differentiation. PLoS One 2014, 9, e89025.

(C) 2014 by the authors; licensee MDPI, Basel, Switzerland. This article is an open access article distributed under the terms and conditions of the Creative Commons Attribution license (http://creativecommons.org/licenses/by/4.0/). 